\title{
ANALYSIS HOLZER METHOD USING QUADRATICPOLYNOMIAL
}

\author{
Tjiang Arif Gunadi
}

Lecturer of Civil Engineering Department, Universitas Kristen Indonesia Paulus, Makassar, Indonesia, Email : tjiangarifgunadi@ukipaulus.ac.id

\begin{abstract}
Calculation structures of the eigen vector and eigen value are not easy, therefore there are many theory for that. One of them is Holzer method, in case of the analysis of Holzer method using qudratic polynomial to find the eigen value shows to get quick calculation, so in this journal shows how get the eigen value. The case study indicates that the Holzer method using quadratic polynomial can be used as method of analysis structures.
\end{abstract}

Keywords: Quadraticpolynomial

Cite this Article: Tjiang Arif Gunadi, Analysis Holzer Method Using Quadraticpolynomial, International Journal of Civil Engineering and Technology, 11(6), 2020, pp. 40-43.

https://iaeme.com/Home/issue/IJCIET?Volume $=11 \&$ Issue $=6$

\section{INTRODUCTION}

\subsection{Multy degree of freedom}

Many kind of eigen values of structures can be calculated.

\subsection{Rayleigh Method}

- Rayleigh quotient.

- Iteration of Rayleigh Method.

\subsection{Holzer Method}

Would be showed in this below.

\subsection{Rayleigh-Ritz Method}

Calculation some important of the all the eigen values of the structures.

\subsection{Jacobi Method}

Matrix calculation to calculate all the eigen values of the structures. 


\section{METHODOLOGY}

In this study, only eigen values is considered.

Procedures of Holzer method calculated the eigen value $\omega$ in trial and error sytem :

The steps is following :

- To define the eigen value $\omega$.

- Give displacement in the top floor $\rightarrow 1$ unit displacement

- Inertia force $\rightarrow F_{1}=m \omega^{2} x$.

- Calculated the friction force, for balancing the inertia force.

- Calculate displacement of each floor $\rightarrow \delta=$ friction force $/ \mathrm{k}$

- Repeat this process until supported, if $\omega$ given true, it mean $\delta=0$.

\section{CASE STUDY}

\subsection{Analysis Holzer method using quadratic polynomial}

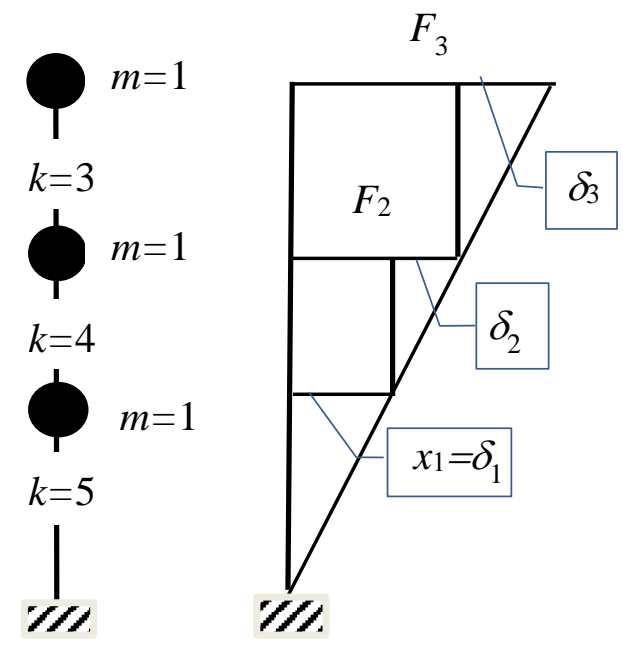

Figure 1 The structure and their mode sharp 1

\section{Top floor}

Inertia force $F_{1}=m \omega^{2} x=1$

Friction force $F_{G}=F_{1}=1$

$\delta_{3}=\frac{\text { friction force }}{\mathrm{k}_{3}}=\frac{1}{3}=0.33$

\section{Second floor}

$x_{2}=x_{3}-\delta_{3}=1-0.33=0.67$

$F_{1}=m \omega^{2} x=1 \times 1 \times 0.67=0.67$

$F_{G}=1+0.67=1.67$

$\delta_{2}=\frac{1.67}{4}=0.42$

\section{First floor}

$x_{1}=x_{2}-\delta_{2}=0.67-0.42=0.25$

$F_{1}=m \omega^{2} x=1 \times 1 \times 0.25=0.25$ 
$F_{G}=1.67+0.25=1.92$

$\delta_{1}=\frac{1.92}{5}=0.38$

Support displacement :

$x_{0}=x_{1}-\delta_{1}=0.25-0.38=-0.13$

For easier calculation, make the table :

Table 1
$\omega^{2}=1$
$\omega^{2}=0,9$
$\omega^{2}=0,8$

$\begin{array}{lll}\delta & \delta\end{array}$

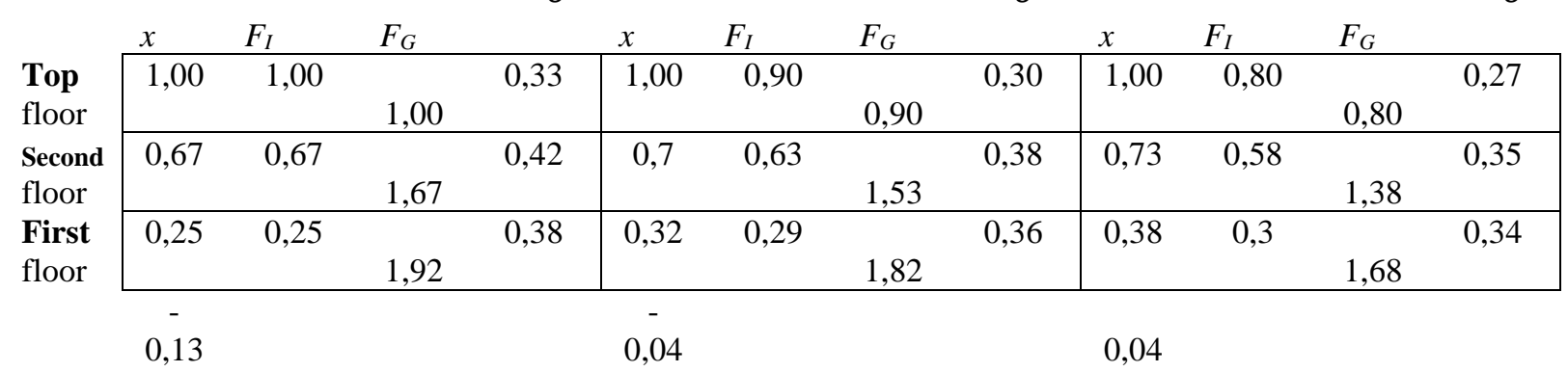

The calculation shows three pairs of numbers $(-0.13,1),(-0.04,0.9)$ and $(0.04,0.8)$.

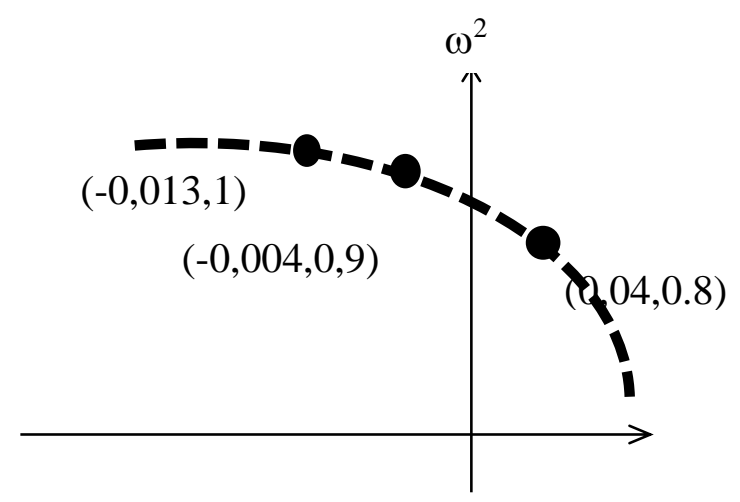

Illustration of quadratic polynomial

Figure 2

If quadratic polynomial $\rightarrow y=a x^{2}+b x+c$

$a=-0.817$

$b=-1.25$

$c=0.851$

$x=0 \rightarrow y=0.851=\omega^{2}$

So the eigen values is 0.851

$\rightarrow$ mode sharp $2 \rightarrow$ cross vertical as 1 time.

same as procedures of the showing procedures 
$\rightarrow$ mode sharp $3 \rightarrow$ cross vertical as 2 time.

same as procedures of the showing procedures

\subsection{Validation}

Using direct calculation :

Table 2

\begin{tabular}{|c|c|c|c|c|}
\hline & \multirow[b]{2}{*}{$x$} & \multicolumn{3}{|c|}{$\omega^{2}=0,851$} \\
\hline & & $F_{I}$ & $F_{G}$ & $\delta$ \\
\hline \multirow{6}{*}{$\begin{array}{l}\text { Top } \\
\text { floor } \\
\text { Second } \\
\text { floor } \\
\text { First } \\
\text { floor }\end{array}$} & 1,00 & 0,851 & & 0,284 \\
\hline & & \multicolumn{3}{|c|}{0,851} \\
\hline & 0,716 & 0,609 & & 0,365 \\
\hline & & \multicolumn{3}{|c|}{1,460} \\
\hline & 0,351 & 0,299 & & 0,352 \\
\hline & & \multicolumn{3}{|c|}{1,759} \\
\hline & $\begin{array}{l}-0,0 \\
\cong 0\end{array}$ & & & \\
\hline
\end{tabular}

\section{CONCLUSION}

The results obtained from case study analysis Holzer method using quadratic polynomial is same with the result from using direct calculation, namely $\omega^{2}=0.851$. This indicates that the analysis Holzer method using quadratic polynomial can be used as method of analysis structures.

\section{REFERENCES}

[1] Applied Technology Council 40, Seismic Evaluation and Retrofit of Concrete Building, California seismic safety commision, 1996.

[2] Badan Standarisasi Nasional (BSN) SNI 2487-2013, Persyaratan beton struktural untuk bangunan gedung, Jakarta, 2013. (Indonesian concrete code same as ACI).

[3] Bathe, Klaus-Jurgen, Finite Element Procedures, Prentis Hall International Edition, New York 07632, 1996.

[4] Chopra, Anil, K, University of California at Berkeley, Dynamics of Structures, Theory and appliacations to earthquike engineering, Third edition, Pearson Prentice Hall, Pearson Education Inc., Upper Sadlle River, New Jersey 07458, 2007.

[5] Clough, Ray W, Penzien, Joseph, Dynamic of Structures, Mc Graw Hill, New York, 1975.

[6] Mac Gregor, James G., Reinforce Concrete (Mechanics and design), Third edition, Prentice-Hall International, Inc, 1988.

[7] Naeim, Farzad, The Seismic Design Handbook, Van nostrand Reinhold, New York, 1989. 